\section{Transtracheal jet ventilation in a porcine model}

\author{
RYAN MCHUGH • MATTHEW KUMAR • JURAJ SPRUNG
}

\author{
RYAN MCHUGH • MATTHEW KUMAR • \\ JURAJ SPRUNG( $\square)$ \\ Mayo Clinic College of Medicine \\ Mary Brigh 2-752, 200 First Street SW \\ Rochester, Minnesota 55905, USA \\ Phone: (507) 255-3298 \\ Fax: (507) 255-6463 \\ E-mail: Sprung.juraj@mayo.edu
}

\begin{abstract}
Hypoxemia is a frequent event during difficult airway management. We propose to use transtracheal jet ventilation (TTJV) early during the management of complex difficult airway scenarios. The objective of this porcine study is to highlight the benefit of oxygenation via prophylactic TTJV. Eighteen pigs (Sus scrofa) were divided into two equal groups. In Group A, pigs were anesthetized and no lung ventilation was conducted following administration of succinylcholine and prior to tracheal intubation. Group B, after induction of anesthesia, received transtracheal ventilation using $100 \%$ oxygen. In both groups intubation was performed after waiting 90 seconds. All intubations were achieved in less than 30 seconds. Postintubation arterial blood gases demonstrated significant hypoxemia in Group $\mathrm{A}\left(\mathrm{PaO}_{2} 22.6 \pm 5.8 \mathrm{~mm} \mathrm{Hg}\right)$, while in Group $B$ oxygenation substantially improved $\left(\mathrm{PaO}_{2} 470.3 \pm 17.0 \mathrm{~mm} \mathrm{Hg}\right)$. The arterial $\mathrm{CO}_{2}$ retention was associated with mild respiratory acidosis ( $\mathrm{pH} 7.26 \pm 0.05)$ in Group A only. These findings prove that prophylactic TTJV can improve oxygenation and allow extra time for definitive management of difficult airway.
\end{abstract}

Keywords: transtracheal catheter; ventilation, tracheal intubation, difficult hypoxemia

\section{Introduction}

During the management of difficult airway, hypoxemia is a major cause of morbidity and mortality. Maxillofacial trauma represents an especially difficult clinical scenario as the combination of airway bleeding, distorted airway anatomy, uncooperative patient and urgency to secure the airway creates a complex situation with limited options. The American Society of Anesthesiologists (ASA) 2003 Difficult Airway Algorithm uses transtracheal jet ventilation (TTJV) as a near last option. (1) We recently reported the prophylactic use of TTJV during the management of difficult airway in two patients with neck and maxillofacial trauma. (2) Early use of TTJV can provide the anesthesiologist with the additional time necessary to prepare for definitive airway manage- ment. This technique should be considered in selective patients who are combative or uncooperative, or who have unusual anatomy, upper airway, facial, and head and neck trauma. Technical details of this procedure can be found in our recent report. (2) Once the jet catheter is placed in the trachea and the oxygen source is attached to the catheter, the patient can be sedated to allow for controlled and less urgent tracheal intubation by the most appropriate technique. In addition to maintaining the patient's oxygenation, TTJV helps the physician in identifying the airway as insufflated oxygen is exiting the glottis creating visible bubbling. In the presence of airway edema, excessive secretions or blood in the airway, this gas escape may help in directing the endotracheal tube during either direct laryngoscopy or fiberoptic bronchoscopy.

The objective of this porcine study is to highlight the benefit of oxygenation via prophylactic TTJV. We propose to initiate early TTJV for two reasons: first, to reduce the hypoxemia, and second, to allow more time for definitive airway management either by surgical means or fiberoptic intubation.

\section{Materials and methods}

With the approval of our Institutional Animal Care and Use Committee, following guidelines established by the National Institutes of Health for the Care and Use of Laboratory Animals, eighteen pigs (species Sus scrofa) weighing 25 to $30 \mathrm{~kg}$ were divided into two groups, Group A and Group B.

Pigs in Group A ( $n=9)$, the control group, were induced with pentobarbital, 300 mg, followed by administration of succinylcholine, $60 \mathrm{mg}$, intravenously (IV). After waiting 90 seconds to simulate a "difficult airway" scenario where ventilation is difficult or impossible, tracheal intubation was performed in less than 30 seconds. In Group B ( $n=9)$, the 
animals received the same induction as pigs in group A. A 12-gauge catheter (Angiocath ${ }^{\circledR}$, Deseret Medical Inc., Park Davis and Company, Sandy, UT) was placed percutaneously across the cricothyroid membrane into the trachea with local anesthesia. Subsequently, Group B pigs received transtracheal ventilation using $100 \% \mathrm{O}_{2}$ at 50 psi at a rate of 15 breaths per minute with an I: E ratio of 1:4 using a manually triggered jet ventilator (Instrumentation Industry, Inc., Bethel Park, PA). Tracheal intubation was performed after waiting 90 seconds. Intubations were achieved in less than 30 seconds. In both groups, arterial blood gases were drawn from pigs before induction and immediately after intubation. Data are expressed as mean + standard deviation. The preintubation and postintubation arterial blood gases were compared using a paired t-test, and the two groups were compared using an unpaired t-test. $A$ value of $P<0.05$ was considered significant.

\section{Results}

Hypoxemia occurred in Group A (without TTJV) at the end of a 90-second period of apnea $\left(\mathrm{PaO}_{2}, 22.6 \pm 5.8 \mathrm{~mm}\right.$ $\mathrm{Hg}$; baseline $\mathrm{PaO}_{2}, 80.5 \pm 4.7 \mathrm{~mm}$ Hg; $P<0.0001$, figure 1). In Group B, oxygenation improved during the 90second period of TTJV and the time required to intubate $\left(\mathrm{PaO}_{2}, 470.3 \pm\right.$ $17.0 \mathrm{mmHg}$; baseline $\mathrm{PaO}_{2}, 83.6 \pm$ $4.2 \mathrm{~mm} \mathrm{Hg}, \mathrm{P}<0.0001$, figure 1). While there was no difference between groups at baseline, the postintubation $\mathrm{PaO}_{2}$ was significantly higher in pigs receiving TTJV $(P<0.0001)$. In both groups, arterial carbon dioxide tension $\left(\mathrm{PaCO}_{2}\right)$ increased (Group A: $\mathrm{PaCO}_{2}, 60.5 \pm$ $4.9 \mathrm{~mm} \mathrm{Hg}$; baseline $\mathrm{PaCO}_{2}, 41.44 \pm$ 3.71 mm Hg; Group B: $\mathrm{PaCO}_{2}, 44.8 \pm$ $3.9 \mathrm{~mm} \mathrm{Hg}$; baseline $\mathrm{PaCO}_{2}, 37.1 \pm$ $1.6 \mathrm{mmHg}$, figure 2). The arterial $\mathrm{CO}_{2}$ retention in Group A was associated with a mild respiratory acidosis $(\mathrm{pH}$ $7.26 \pm 0.05)$.

\section{Discussion}

The exact incidence of hypoxemia during the management of difficult airway is not known but is presumed to be high. Mateer et al. studied emergency room endotracheal intubations and reported a $10.4 \%$ incidence of hypoxemia. (3) During difficult intubation the incidence of hypoxemia is likely to be even greater.
In our study, we found a large decrease in arterial oxygenation after a 2-minute apnea; this hypoxemia was associated with hypercapnia $(61 \mathrm{mmHg})$ and respiratory acidosis $(\mathrm{pH} 7.26)$ (figures $1,2)$. In contrast, in animals receiving

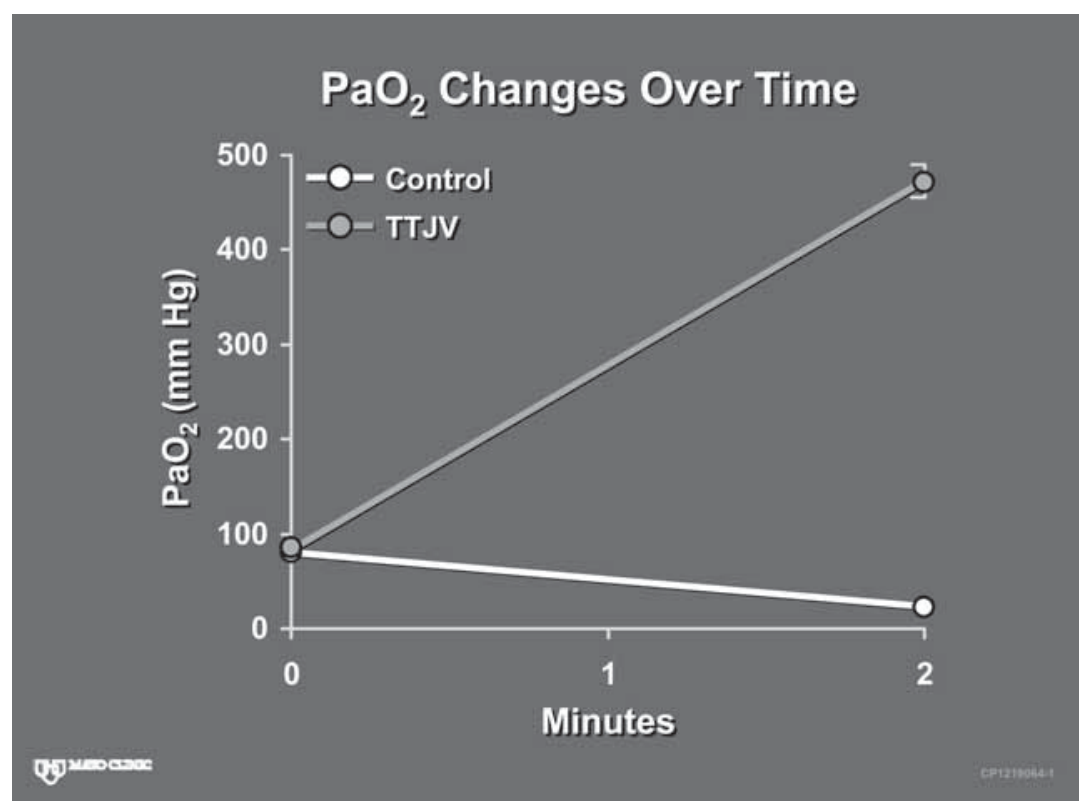

Figure 1. Control group (nonventilated pigs) became profoundly hypoxemic during apnea. Pigs in TTJV group had improvement in oxygenation during the 2-minute period before tracheal intubation $(P$ values were $<0.0001)$.

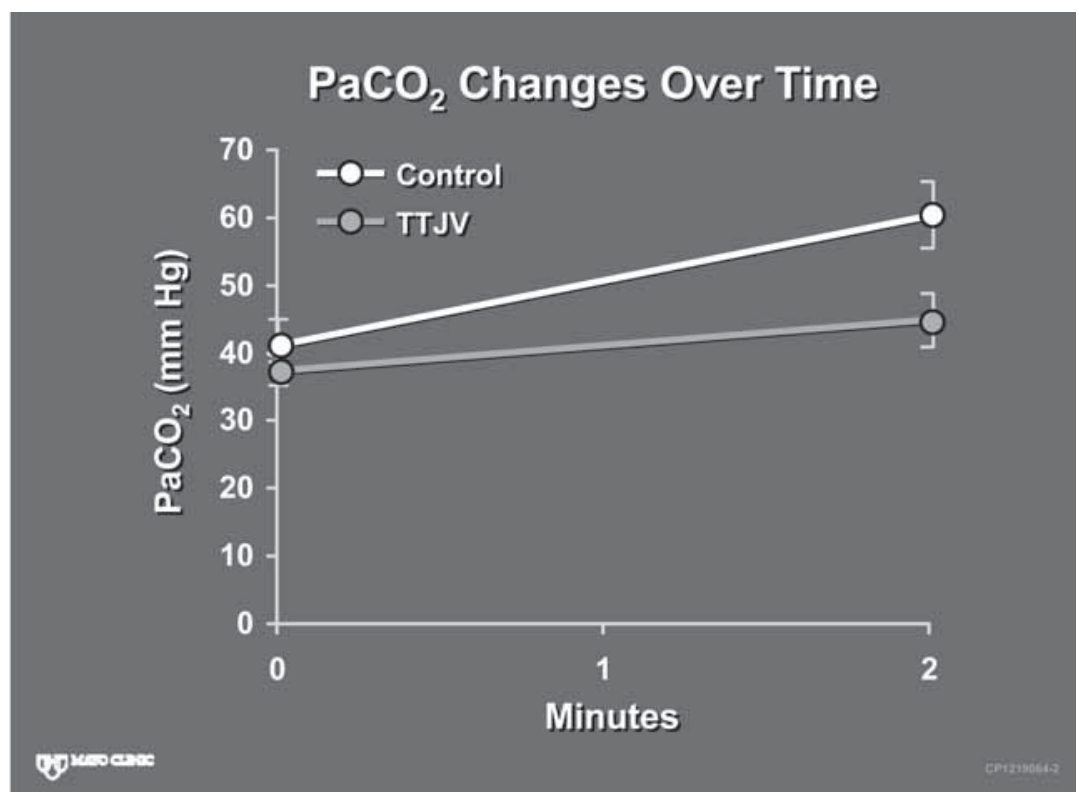

Figure 2. In control and TTJV groups, arterial carbon dioxide tension (PaCO2) increased during the 2-minute period, more so in non-ventilated animals (controls). 


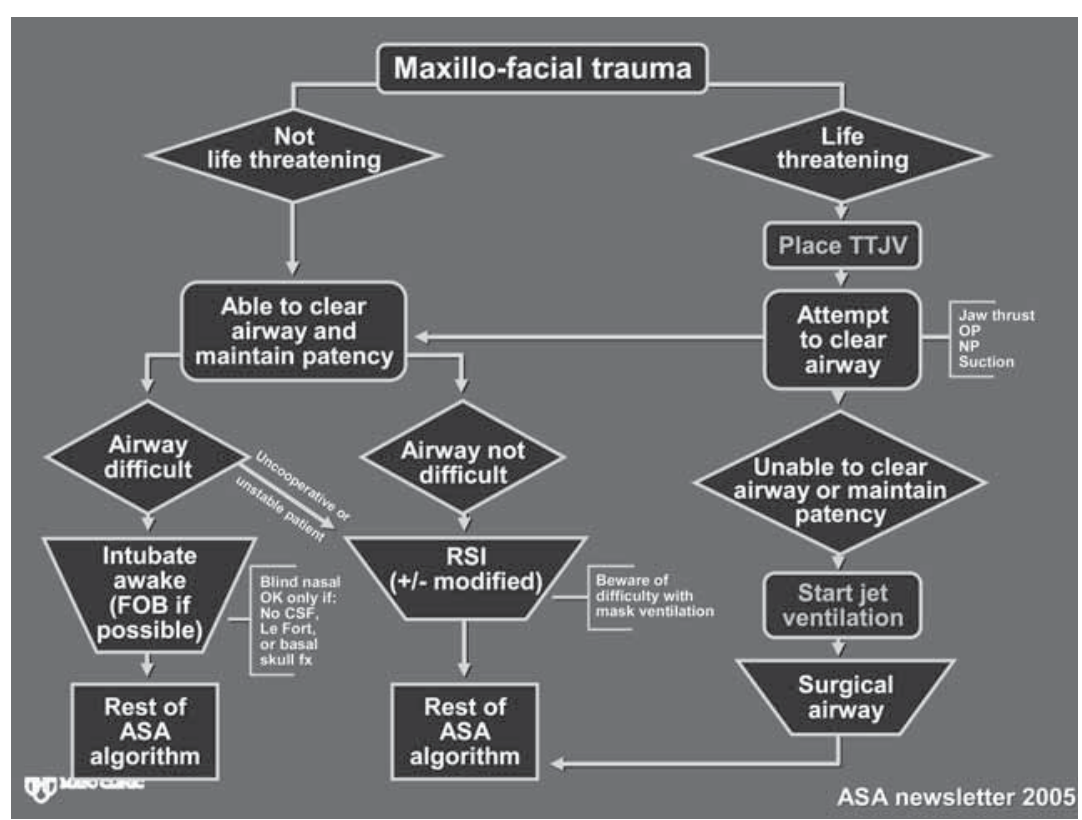

Figure 3. Maxillofacial trauma algorithm with the two modifications we propose noted in orange lettering. This flow diagram highlights a subgroup of patients where prophylactic TTJV may be of the most benefit. This algorithm divides management into life-threatening versus non life-threatening. In non life-threatening scenarios it may not be appropriate to use prophylactic TTJV. However, in life-threatening situations where airway access is required, prophylactic TTJV can be placed safely prior to sedation and loss of airway reflexes. When airway is unable to be established, then jet ventilation should be initiated while awaiting surgical intervention. Used with permission from ASA newsletter. (10)

TTJV, oxygenation drastically improved while hypercapnia remained at modest level $(45 \mathrm{mmHg})$ during the same time period. Thus, early TTJV prevents hypoxemia and provides the anesthesiologist with extra time to provide sedation, perform tracheal intubation or gain surgical airway. While arrangements for the definitive management of the difficult airway (fiberoptic intubation, surgical airway) may require some preparatory time, a tracheal catheter for jet ventilation can be inserted quickly and atraumatically with readily available equipment such as 12- or 14-gauge angiocatheters. (2)
Several studies have evaluated the use of TTJV in animal models. (4-7) Recently, Schaefer et al. demonstrated sufficient oxygenation with TTJV in a porcine model with a self-made airway device similar to the one used in this study. (7) Furthermore, clinical use of TTJV during the management of difficult airway has been reported. $(2,8,9)$ We recently reported the usefulness of TTJV placed prophylactically in two patients with neck and maxillofacial trauma. (2) Early use of TTJV allowed us to maintain adequate oxygenation in these uncooperative and critically injured patients and permitted more time for securing the airway with fiberoptic intubation. Figure 3 depicts a modified maxillofacial trauma algorithm (10) and demonstrates the position within this algorithm we propose for preemptive use of TTJV in scenarios of complex difficult airway. TTJV may be equally helpful in gaining additional time while preparing for other options for invasive airway access (percutaneous tracheostomy and cricothyroidotomy).

The use of TTJV is not without complications. One of the most frequently described complications is dislodgement of the catheter during TTJV which may result in subcutaneous emphysema. (11) This potentially catastrophic situation can convert the difficult airway into an "impossible airway." Other complications of TTJV include bleeding, pneumothorax, barotrauma, tracheal and esophageal perforation as well as lifethreatening intravascular misplacement of the catheter. (12-14) In addition, when TTJV was placed emergently instead of prophylactically, complications were twice as likely to occur. (11) In order to avoid these complications it is of the utmost importance to check the proper position of the catheter before the initiation of jet ventilation by confirming the ability of free-air aspiration with a large syringe. It is equally important to have a dedicated assistant who will keep the catheter secure in the position and who will be monitoring for signs of dislodgement (subcutaneous emphysema).

In conclusion, we have demonstrated in a porcine model that the prophylactic use of TTJV by using a simple, readily available set-up is an efficient method for maintaining arterial oxygenation. This allows extra time for intubation of the trachea either by bronchoscope or direct laryngoscopy during "cannot intubate, cannot ventilate" scenarios or in patients with difficult airway due to head or neck trauma. 


\section{REFERENCES}

1. Practice guidelines for management of the difficult airway: an updated report by the American Society of Anesthesiologists Task Force on Management of the Difficult Airway. Anesthesiology 2003;98:1269-77.

2. McHugh R, Kumar M, Sprung J, Bourke D. Transtracheal jet ventilation in management of the difficult airway. Anaesth Intensive Care 2007;35:406-8.

3. Mateer JR, Olson DW, Stueven HA, Aufderheide TP. Continuous pulse oximetry during emergency endotracheal intubation. Ann Emerg Med 1993;22:675-9.

4. Frame SB, Timberlake GA, Kerstein MD, Money MK, Hendrickson MF, Akers DL, et al, editors. Transtracheal needle catheter ventilation in complete airway obstruction: an animal model. Ann Emerg Med 1989;18:127-33.

5. Hauswald M, Ong G, Yeoh E. Percutaneous needle cricothyroidotomy with repetitive airway obstruction. Am J Emerg Med 1995;13:623-5.

6. Preussler NP, Schreiber T, Huter L, Gottschall R, Schubert H, Rek H, et al, editors. Percutaneous transtracheal ventilation: effects of a new oxygen flow modulator on oxygenation and ventilation in pigs compared with a hand triggered emergency jet injector. Resuscitation 2003;56:329-33.

7. Schaefer R, Hueter L, Preussler NP, Schreiber T, Schwarzkopf K. Percutaneous transtracheal emergency ventilation with a self-made device in an animal model. Paediatr Anaesth 2007;17:972-6.

8. Benumof JL, Scheller MS. The importance of transtracheal jet ventilation in the management of the difficult airway. Anesthesiology 1989;71:769-78.

9. Patel RG. Percutaneous transtracheal jet ventilation: a safe, quick, and temporary way to provide oxygenation and ventilation when conventional methods are unsuccessful. Chest 1999;116:1689-94.

10. Wilson WC. Trauma: Airway management. ASA difficult algorithm modified to trauma--and five common trauma intubation scenarios. ASA Newsletter 2005;69:9-16.

11. Smith RB, Schaer WB, Pfaeffle H. Percutaneous transtracheal ventilation for anaesthesia and resuscitation: a review and report of complications. Can Anaesth Soc J 1975;22:607-12

12. Bourgain JL, Desruennes E, Fischler M, Ravussin P. Transtracheal high frequency jet ventilation for endoscopic airway surgery: a multicentre study. Br J Anaesth 2001;87:870-5.

13. Chang JL, Bleyaert A, Bedger R. Unilateral pneumothorax following jet ventilation during general anesthesia. Anesthesiology 1980;53:244-6.

14. Weymuller EA, Jr., Pavlin EG, Paugh D, Cummings CW. Management of difficult airway problems with percutaneous transtracheal ventilation. Ann Otol Rhinol Laryngol 1987;96:34-7. 